\title{
Thermodynamics and Kinetics in Antibody Resistance of the 501Y.V2 SARS-CoV-2 Variant
}

\author{
Son Tung Ngo, ${ }^{\text {ab* }}$ Trung Hai Nguyen, ${ }^{\text {ab }}$ Duc-Hung Pham, ${ }^{\mathrm{c}}$ and Nguyen Thanh Tung, ${ }^{\text {de }}$ Pham Cam \\ Nam, ${ }^{* *}$ \\ Understanding thermodynamics and kinetics of the binding process of an antibody to SARS-CoV-2 receptor-binding domain \\ (RBD) of Spike protein is very important for the development of COVID19 vaccines. Especially, it is essential to understand \\ how the binding mechanism may change under the effects of RBD mutations. In this context, we have demonstrated that \\ the South African variant (B1.351 or 501Y.V2) can resist the neutralizing antibody (NAb). Three substitutions in RBD including \\ K417N, E484K, and N501Y alters the free energy landscape, binding pose, binding free energy, binding kinetics, hydrogen \\ bond, nonbonded contacts, and unbinding pathway of RBD + NAb complexes. The low binding affinity of NAb to 501Y.V2 \\ RBD confirms the antibody resistance of the South African variant. Moreover, the fragment of NAb + RBD can be used as an \\ affordable model to investigate the change of the binding process between mutations RBD and antibodies.
}

\section{Introduction}

The novel $\beta$-coronavirus, SARS-CoV-2, whose sequence is similar to SARS-CoV-1 and MERS-CoV inducing human respiratory epidemic at the beginning of this century, is the cause of the human respiratory disease (COVID-19) pandemic worldwide. ${ }^{1,2}$ This virus has been infecting more than 160 million cases and associated with more than 3 million deaths. ${ }^{3}$ SARS-CoV-2 is a single-positive-strand RNA virus, whose genome encodes for four main components: spike, envelope, membrane and nucleocapsid. ${ }^{4,5}$ The spike protein (S protein) of SARS-CoV-2 which is used by the virus to bind to human angiotensinconverting-enzyme 2 (ACE2), has been researched thoroughly. ACE2 is present in different tissues in the body, including the lung, heart and liver, ${ }^{6}$ is employed by SARS-CoV- 2 as receptor to bind and infect human cells. The $S$ trimer comprises three copies of $S 1$ and $S 2$ subunits. S1 subunit contains 4 domains: S1A, S1B, S1C and S1D, in which S1B domain is also called receptor-binding domain (RBD), which mediates the attachment of spike protein to target cell via binding to ACE2 receptor. ${ }^{7}$ Once RBD is in the 'up' conformation, it can recognize and bind into ACE2, which leads to the conformational changes of S2 subunit and enables SARS-CoV-2 to fuse with cell membrane and to enter host cells. ${ }^{1,7}$

RBD is the main target of neutralizing antibodies (NAbs) which can be isolated from plasma of COVID-19 patients, immunoglobulin libraries, or immunized laboratory animal models. ${ }^{1}$ These NAbs can be roughly divided into four main classes, of which class $1 s^{\prime}$ and class $2 s^{\prime}$ RBD epitopes overlap with the ACE2-binding site, suggesting a neutralization mechanism that involves direct competition with ACE2. Class 1 antibodies, which are encoded by the immunoglobulin V-gene (VH3-53) segment with complementarity-determining regions 1 and 2 (CDRH1 and $C D R H 2$ ) and a short CDRH3, are mostly elicited by SARS-CoV-2 infection. On the other hand, when class 2 antibodies also target site I10,15 which is also target epitopes of class 1 antibodies, they bind to RBD in both 'up' and down' conformations of $S$ protein. ${ }^{1,8}$ Additionally, class 3 antibodies bind outside ACE2 and recognize both up and down RBD, while class 4 antibodies comprise previously described antibodies that cannot block ACE2 and target only to RBD in 'up' conformation. ${ }^{1}$ Besides RBD, the $\mathrm{N}$-terminal domain (NTD) of protein $\mathrm{S}$ is also a popular target for NAbs and many potent monoclonal antibodies directed against this region show great potential in clinical trials for COVID-19 treatment. ${ }^{8}$ The majority of these antibodies target a single immunodominant site on NTD, including the N1-loop (NTD N-terminus), N3-loop (supersite b- hairpin), and N5 loop (supersite loop). Subsets of these antibodies and NAbs in class 1 and class 3 form multi-donor classes, with different set of $\mathrm{VH}$ germline restricted mode of spike recognition. ${ }^{8}$

Due to many reasons, including high transmissibility, the longevity of the pandemic, and encountering with immunocompromised hosts, SARS-CoV-2 undergoes different rounds of mutations, which has altered the structures of the virus, modulated its infectivity, and changed the antigenicity of the surface proteins. ${ }^{9}$ The variants, including United Kingdom (B1.1.7) and South African (B1.351 or 501Y.V2) variants have associated with increased transmissibility and possibly increased mortality. ${ }^{8}$ Especially, the SARS-CoV-2 lineage in South Africa, included nine mutations in the spike protein, seems to decrease the efficacy of NAb as well as Covid-19 vaccine efficacy of some vaccines currently being used. ${ }^{10,11}$ The mutations in B1.351 can be divided into two groups, one concentrates in NTD, including four substitutions and a deletion (L18F, D80A, D215G, $2242-244$, and R246I), and the other involves three substitutions in RBD (K417N, E484K, and N501Y). ${ }^{12}$ These changes induce S protein biological and structural alterations. Especially, mutation E484K is very critical, which can reduce the effect of NAb. ${ }^{13}$

Evaluating antibody resistance of the 501Y.V2 SARS-CoV-2 variant is greatly attractive to scientists. ${ }^{8}, 10,11,14$ Understanding the physical insights into the process probably enhances the vaccine developments, but the knowledge is still limited. Therefore, in this context, atomistic simulations were carried out to reveal the insights at the atomic level of the binding process of NAb to 501Y.V2 SARSCoV-2 RBD. For the first step, structural changes of the 501Y.V2 and wildtype (WT) SARS-CoV-2 RBD + NAb complexes were characterized via unbiased MD simulations. Thermodynamics and kinetics of the binding process were then revealed via biased MD simulations. Moreover, fragment of NAb (fNAb) is often used to study the binding of $S$ protein/RBD to antibody. ${ }^{15,}{ }^{16}$ In this work we also investigated binding of fNAb (cf. Figure $1 E$ ) to RBD to evaluate the possibility of using fNAb in studying the influence of RBD mutations on the binding affinity instead of using NAb which is a larger molecule and costs more computing resources. Furthermore, it should be noted that glycosylation of RBD was neglected to clarify the interaction nature between RBD + antibodies, although glycans play an important role in the modulation of the spike conformational dynamics. ${ }^{17-19}$ Details of simulations were described in Figure 1 and the Electronic Supplementary (ESI) file. 


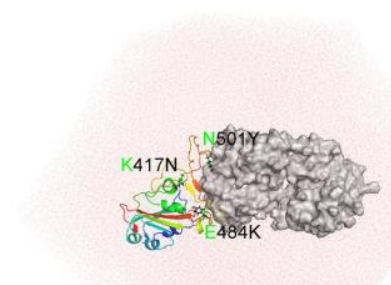

(A)
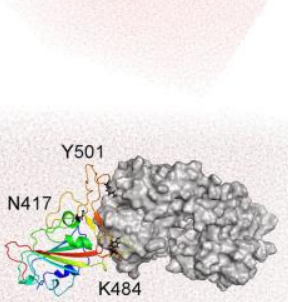

(C)

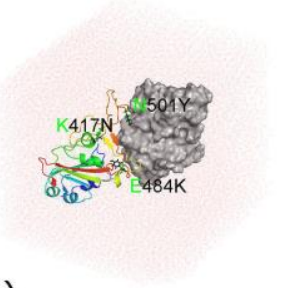

(B)

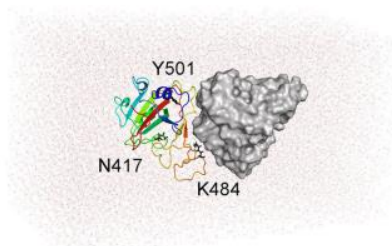

(D)

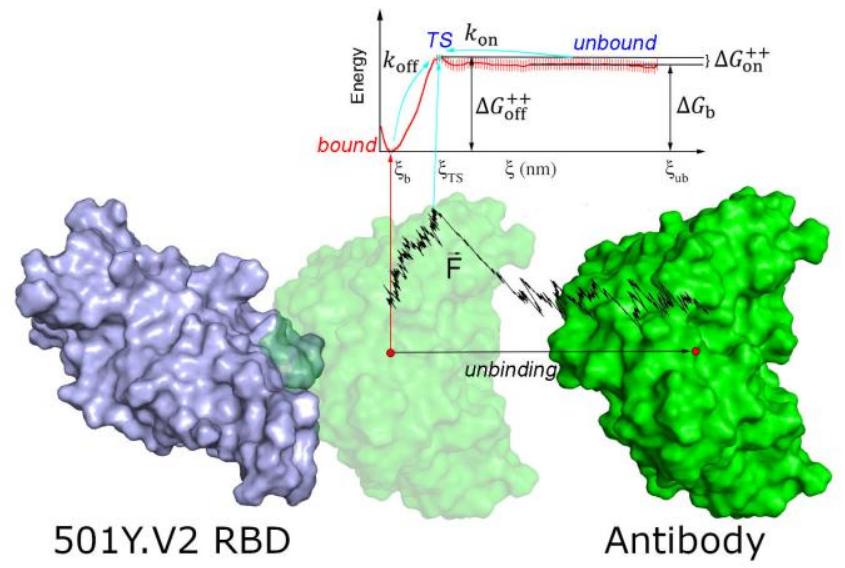

(E)

Figure 1. Starting structures of RBD + antibody systems. (A) $501 Y . V_{2}$ RBD + NAb in MD simulations; (B) $501 Y . V_{2}$ RBD + fNAb in MD simulations; (C) + (D) $501 Y . V_{2}$ RBD + $\mathrm{NAb} / \mathrm{fNAb}$ in biased MD simulations; (E) Free energy scheme. The WT RBD + antibody complexes were formed an initial conformation similar to the 5oiY.V2 one. The fNAb was mobilized from bound to unbound states via FPL calculations, then the free energy profile was calculated via US simulations.

\section{Computational Methods}

\section{Structure of SARS-CoV-2 Antibodies and RBD}

The three-dimensional structure of SARS-CoV-2 RBD and their antibodies NAb was found from the Protein Data Bank (PDB) with the identities of 7BWJ. ${ }^{20}$ The fNAb was extracted from NAb as showed in Figure 1E. The resolution of $7 \mathrm{BWJ}$ and $2.85 \AA$. Moreover, as mentioned above, it should be noted that the new variant of the SARS-CoV-2 in South Africa, B1.351 or 501Y.V2, forms eight changes in the Spike protein. There are four substitutions and a deletion in the $\mathrm{N}$-terminal domain (NTD) including L18F, D80A, D215G, $4242-244$, and R246I. Consequently, three substitutions were found in RBD involving K417N, E484K, and N501Y. The RBD structure with three substitutions was thus prepared via changing three residues of 7BWJ using the PyMOL mutagen tools. ${ }^{21}$

\section{Molecular Dynamics Simulations}

The atomistic simulation was performed using the GROMACS version 5.1.5 with general-purpose computing on graphics processing units. ${ }^{22}$ The protein, antibody, and neutralized ions were parameterized via the Amber99SB-iLDN force field ${ }^{23}$ since it is one of the most suitable force fields for free energy calculation. ${ }^{24,25}$ The TIP3P water model was chosen to simulate the water molecule. ${ }^{26}$ The systemic configurations were shown and detailly reported in Figure $1 \mathrm{~A}-\mathrm{B}$ and Table $\mathrm{S} 1$ of the ESI.

The MD simulation parameters were referred to the previous works. ${ }^{27,} 28$ However, in particular, the integral was efforted every 3 femtoseconds. A non-bonded pair between two atoms were affected within a radius of $0.9 \mathrm{~nm}$, in which the electrostatic interaction was calculated using the fast Particle-Mesh Ewald electrostatics approach $^{29}$ as well as the van der Waals (vdW) interaction was computed using the cut-off scheme. The solvated complex was initially optimized using the energic minimization simulation via the steepest descent method. The minimized system was then relaxed in NVT and NPT ensembles with a length of 100 ps each simulation. During NVT and NPT simulations, the integral was attempted every 1 femtosecond. The equilibrium snapshots obtained via NPT simulations were used as starting conformations of MD simulations.
The conventional MD simulations were performed with interval 100 ns and repeated 4 times independently.

\section{Biased Molecular Dynamics Simulations}

Steered-MD simulation. Representative structures of RBD + antibody systems, which were obtained via MD simulations, were employed as initial shapes of FPL simulations. The complexes were reinserted into the rectangular PBC box for saving the computing resources. The configuration information was described in Figure 1CD and Table S1. The FPL simulations were carried out to generate unbinding conformations of the systems, which were used as starting shapes of US simulations. From the beginning, the antibodies were forced to dissociate from the binding mode with the WT/501Y.V2 RBD using SMD simulations. In particular, eight SMD trajectories were carried out to probe the most optimal-unbinding pathway. The trajectory, in which the rupture force, $F_{\mathrm{Max}}$, and pulling work, $W$, formed the smallest deviation in comparison with the median values, was used for generating US windows. In FPL, the antibody was pulled along Z-axis via an external force using cantilever $k=1000 \mathrm{~kJ} \mathrm{~mol}^{-1}$ $\mathrm{nm}^{-2}$ and constant velocity $v=0.001 \mathrm{~nm} \mathrm{ps}^{-1}$. During the simulation, the RBD was softly fixed via $C_{\alpha}$ restraint. The pulling force, antibody displacement, and systemic coordinates were recorded every 33 integrated steps.

Umbrella sampling simulation. The systemic snapshots, which were extracted from the FPL trajectory since the antibody displaced every ca. $1.0 \AA$ along the unbinding pathway $\xi$, were used as starting shapes of US simulations. Ca. 25 US windows each complex were simulated with a length of $10 \mathrm{~ns}$ of MD simulation to calculate the potential of mean force (PMF) curve. It should be noted that a short NPT simulation was executed to reduce initial fluctuations. ${ }^{30,31}$ The PMF values were calculated via the weighted histogram analysis method (WHAM). ${ }^{32}$ The free energy barriers, $\Delta G_{\text {on }}^{++}$and $\Delta G_{\text {off }}^{++}$, and binding free energy, $\Delta G_{\mathrm{b}}$, of the binding process between RBD and NAb were estimated as described as Figure $1 \mathrm{E}$.

\section{Analyzed Tools}

The free energy landscape (FEL) of the complex was constructed using the principal component analysis (PCA) method, ${ }^{33}$ in which coordinates $\mathrm{CV} 1$, first eigenvector, and $\mathrm{CV} 2$, second eigenvector, were calculated using GROMACS tools "gmx anaeig". A non-bonded (NB) contact was counted when the pair between two heavy atoms 
is smaller than $4.5 \AA$. A hydrogen bond (HB) contact was counted when the angle $\angle$ between donor $(D)$ - hydrogen $(H)$ - acceptor $(A)$ is larger than $135^{\circ}$ and the distance between $D$ and $A$ is smaller than 3.5 ̊. The PMF value was estimated via the Weighted Histogram Analysis Method (WHAM) with the execution of auto-correlated time. The computed error was calculated using the bootstrapping method. ${ }^{34}$ The solvent accessible surface area (SASA) was computed using GROMACS tool "gmx sasa".

\section{Results and Discussion}

It should be noted that investigating structures of protein-protein complexes and understanding how they bind together are fundamental issues. ${ }^{35}$ Moreover, structures of several complexes remain difficult to solve experimentally. ${ }^{36,37}$ Furthermore, in order to characterize the protein-protein binding mechanisms, powerful experimental approaches are required, ${ }^{38,39}$ but the obtained data are normally limited or indirect. Obtaining direct data at an atomic level about binding pathways and physical insights into the binding mechanisms are still open issues..$^{35}$ Atomistic MD simulations emerge as potential approaches for investigating both dynamics and structural change of protein-protein complexes. ${ }^{17,} 40,41$ Using MD simulations, we can easily monitor the associate and dissociate processes of a monomer to the others. ${ }^{28,}{ }^{42}$ However, in fact, the association of two proteins may take place at a much longer time scale than unbiased simulations can usually reach. Normally, the enhanced sampling methods, which may combine several short simulation trajectories, are used to modeling the unbinding process of two proteins. ${ }^{30,43}$ The association of protein-protein is thus predicted..$^{43}$ Therefore, in this work, atomistic simulations will be performed to reveal the insights at the atomic level of the binding process of an antibody to various SARS-CoV-2 variants RBD.
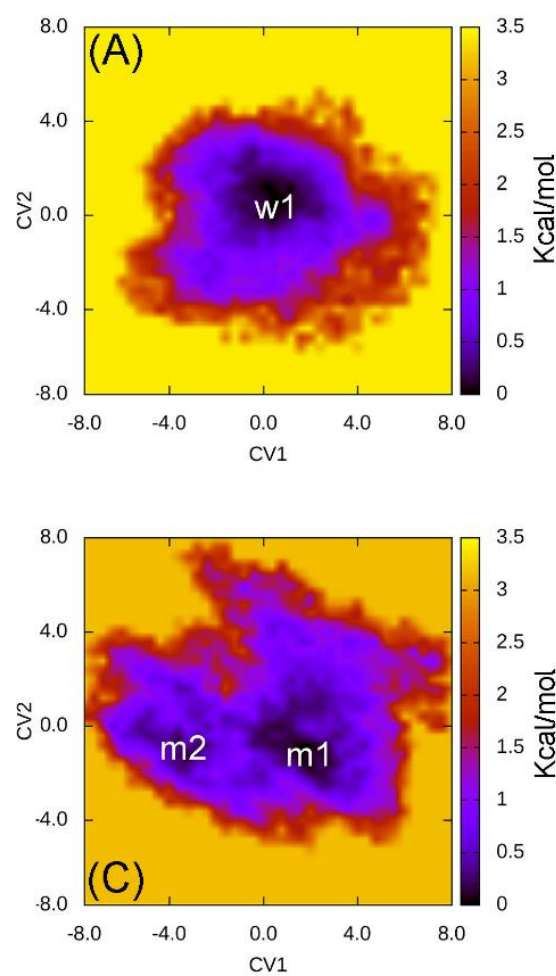

Structural changes of the SARS-CoV-2 RBD + antibody complexes were characterized via MD simulations. Thermodynamics and kinetics of the binding process were then revealed via a combination of SMD and US simulations. The obtained results probably enhance the vaccine developments.

Unbiased MD simulations were carried out to understand the structural change at the atomistic level of 501Y.V2 RBD + antibodies since the binding affinity of the antibodies to 501Y.V2 RBD was altered according to the recent report. ${ }^{8}, 10,11,14$ The stabilized conformations of the RBD + antibody complexes were investigated over the equilibrium trajectories (cf. Figure S1 of the ESI file). The structural changes of the complexes were reported in Figures S2-S3 of the ESI file. In particular, the backbone root-mean-square deviation (RMSD) and SASA of the complexes were enlarged when the 501Y.V2 variant were induced. However, the HB and NB contacts between RBD and NAb/fNAb were significantly reduced due to mutations. The obtained results imply that the protein-protein binding affinity between 501Y.V2 RBD to NAb/fNAb was decreased in comparison with WT one.

In order to estimate the representative conformations of the complexes, the two-dimensional FEL was generated using "gmx sham" tool. ${ }^{33,44}$ Two coordinates constructing FEL were first and second eigenvectors, which were computed using the principal component analysis (PCA) method. ${ }^{33}$ The obtained results were described in Figure 2. Clearly, the 501Y.V2 variant increases the number of the FEL local minima implying that the 501Y.V2 complex is more flexible than the WT one. It also suggests that the binding free energy $\Delta G_{\mathrm{b}}$ between 501Y.V2 RBD and NAbs is probably reduced. The obtained results are in good consistent with NB and HB contacts analyses above.
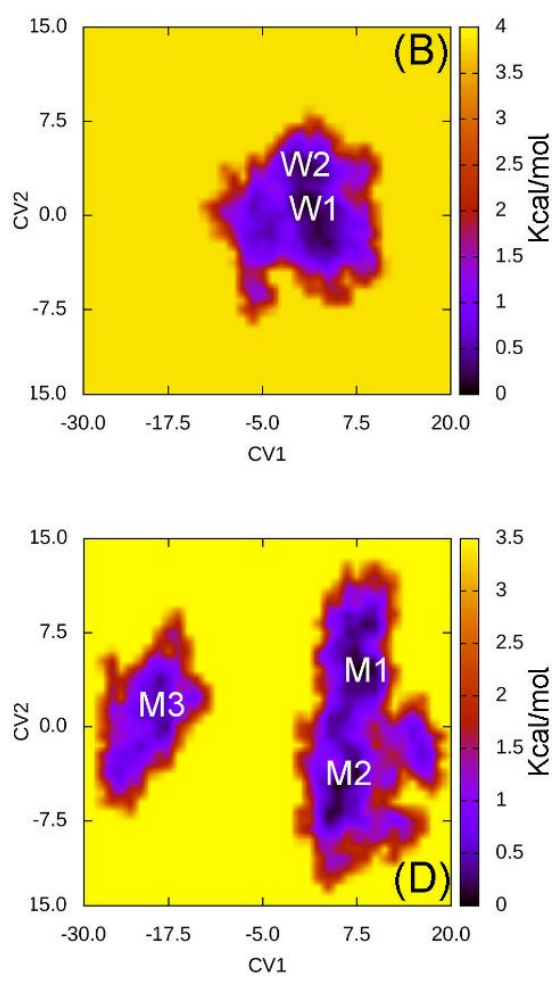

Figure 2. Free energy landscape of RBD + antibody complexes was constructed using PCA method. In particular, (A) presents the FEL of the WT RBD + fNAb; (B) mentions the FEL of the WT RBD + NAb; (C) describes the FEL of the $501 Y . V_{2}$ RBD + fNAb; and (D) denotes the FEL of the $501 Y . V_{2}$ RBD + NAb.

The WT RBD + fNAb only formed one minimum noted as w1 in Figure $2 \mathrm{~A}$, which is located at (CV1; CV2) coordinates of $(0.40 ; 0.40)$.
In particular, the antibody adopted HBs to 4 residues of the WT RBD including G447, Y449, N450, and E484 (cf. Figure 3). These results 
suggest that a mutation E484K will significantly alter the binding affinity/mechanism of the RBD + fNAb. Two minima were observed in FEL of 501Y.V2 RBD + fNAb, which are located at (CV1; CV2) coordinates of $(1.60 ;-1.40)$ and $(-3.60 ;-1.00)$ denoted as $\mathbf{m} \mathbf{1}$ and $\mathbf{m} \mathbf{2}$, respectively. Analyzing the representative structure $\mathbf{m} 1$, the antibody was found to be able to form HBs to the residues K444,
G447, Y449, and N450 of the 501Y.V2 RBD. The corresponding residues of $\mathbf{m} 2$, which formed HBs to RBD 2-4, are G447, Y449, N450, and K484 (cf. Figure 3). The observed structural changes imply that the binding affinity and kinetics between RBD and fNAb probably change. A similar story of RBD + NAb, which is mentioned in detail in the ESI file, was obtained and confirmed the results.

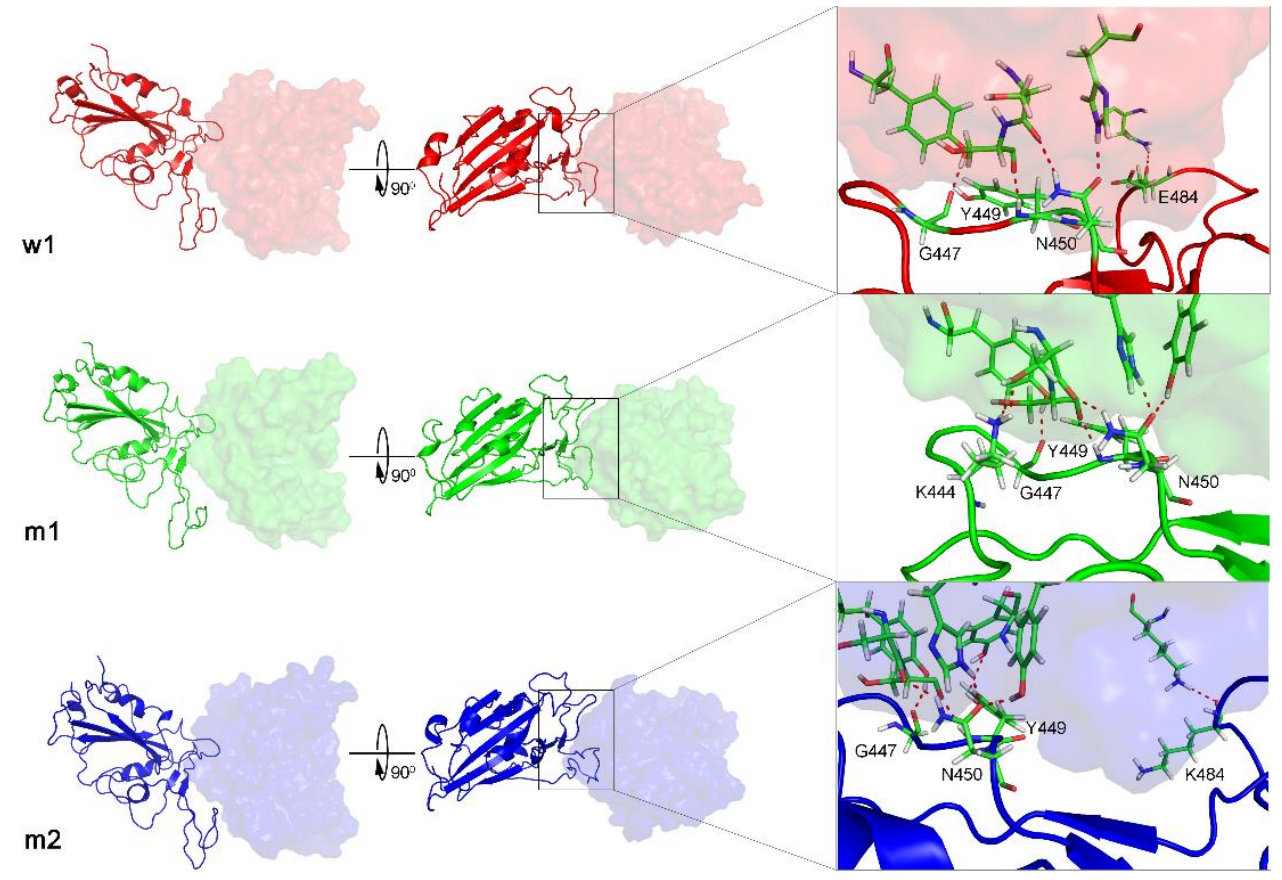

Figure 3. The representative structures of WT and 501Y.V2 RBD + fNAb in different perspective. The structures corresponds to the minima w1, mi, and mz.

The WT/501Y.V2 RBD + NAb systems were also investigated. FEL of the complexes was significantly altered when the mutations were induced. The WT RBD + NAb formed two minima, which were shown in Figure 2B. These minima located at (CV1; CV2) coordinates of $(0.63 ; 0.75)$ and $(0.63 ; 3.38)$ denoting as W1 and W2, respectively. Besides that, the 501Y.V2 $\mathrm{RBD}+\mathrm{NAb}$ FEL (Figure 2D) adopted three minima, which located at (CV1; CV2) coordinates of (7.50; 4.13), (5.63; -4.50), and $(-18.8 ; 1.50)$ labelling as $\mathbf{M} \mathbf{1}, \mathbf{M} 2$, and $\mathbf{M} 3$, respectively. Analyzing the complex W1, the HBs were observed between antibody and residues G447, Y449, N450, and E484 of the RBD that is in good consistency to the w1 case. However, HBs were only found between the NAb and residue E484 of the WT RBD in the complex W2 (Figure 4). The obtained results indicate that residue $\mathrm{E} 484$ plays an important role in the binding process of the antibody to the RBD that is in good consistent with the recent work. ${ }^{13}$ Replacing the E484 with another residue probably modifies the binding mechanism of the antibodies to RBD rather than substitutions at the different positions. Moreover, it should be noted that in the 501Y.V2 variant induced, a lysine residue substitutes the glutamate residue at the sequence 484 . The replacement probably terminates the $\mathrm{HBs}$ and weakening the attracted force between the NAb and the RBD. The argument was confirmed via evaluations of the representative structures of 501Y.V2 RBD + NAb complexes. In conformation M1, the HBs between NAb and the residues $\mathrm{G} 447$, Y449, and N450 of RBD were found. The residues G447, Y449, $\mathrm{N} 450$, and T470 of 501Y.V2 RBD procedure HBs to NAb in conformation M2. Furthermore, The NAb only found two HBs to the residue E471 and N481 of the 501Y.V2 RBD. The free energy approach should be carried out to clarify the change of binding affinity upon the structural changes of the 501Y.V2 RBD + NAb complexes. As discussed above, the RBD + fNAb structure is more flexible when the 501Y.V2 variant was induced. The binding affinity/mechanism of the complex is thus altered. In this work, a combination of steered-molecular dynamics (SMD)/umbrella sampling (US) simulations were carried out to probe the change in RBD + NAbs association. The SMD was used to generate US windows (cf. the ESI file). The free energy profile was then calculated using the WHAM. ${ }^{32}$ The binding free energy $\Delta G_{\mathrm{b}}$ between RBD and NAbs is able to calculate via PMF curve as mentioned in Figure 1E. ${ }^{30}, 31$ Moreover, the free energy barriers $\Delta G_{\text {on }}^{++}$and $\Delta G_{\text {off }}^{++}$, which were associated with the binding kinetic rate constant $k_{\text {on }}$ and the unbinding kinetic rate constant $k_{\text {off }}$ can be also estimated, respectively. 


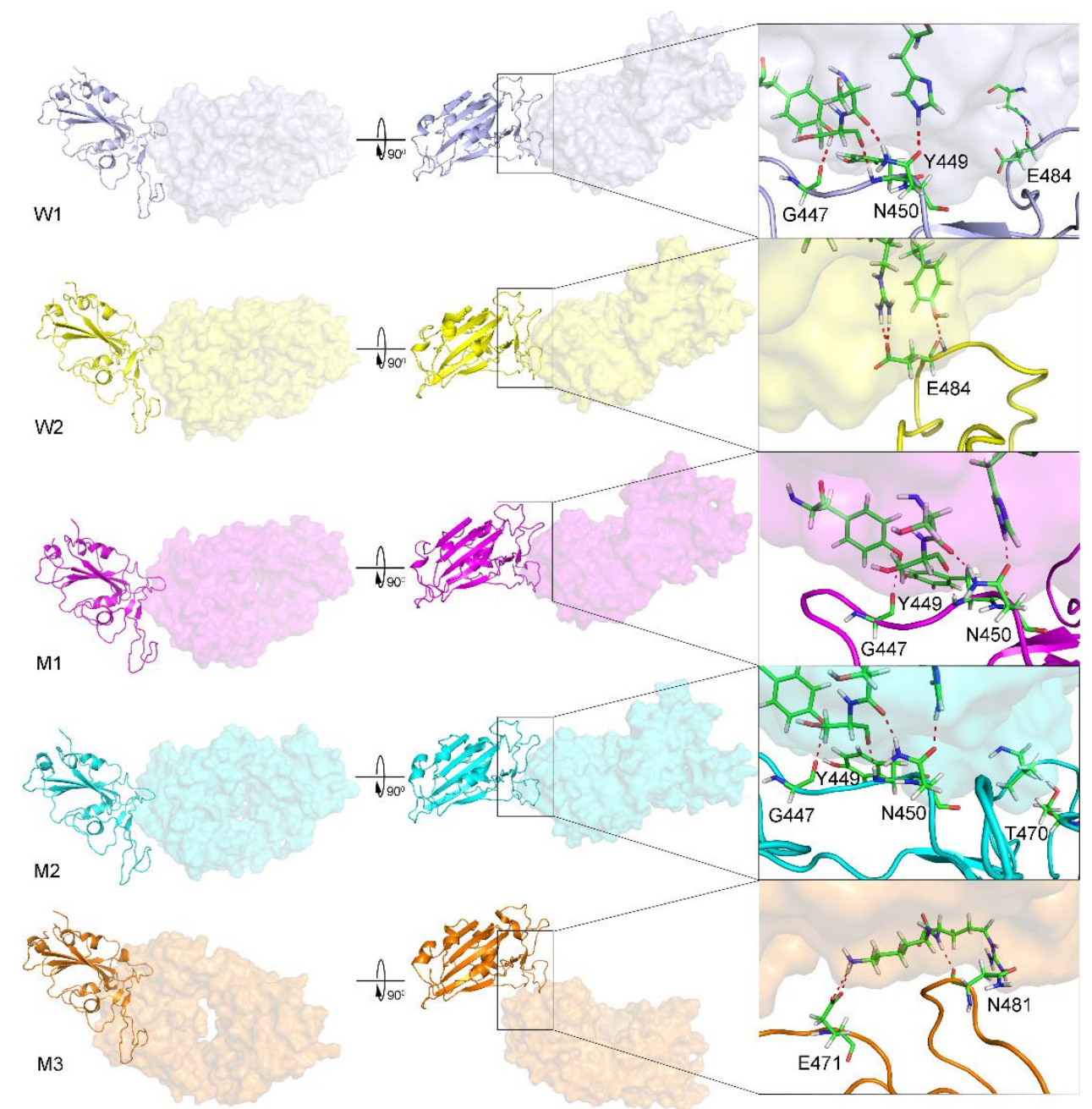

Figure 4. The representative structures of WT and 501Y.V2 RBD + NAb corresponding to the minima W1, W2, M1, M2, and M3. The interaction diagram between RBD and NAb were obtained using PyMOL tool.

As discussion above, the RBD + fNAb structure is more flexible when the 501Y.V2 variant was induced. The binding affinity/mechanism of the complex are thus altered. In this work, a combination of steered-molecular dynamics (SMD)/umbrella sampling (US) simulations were carried out to probe the change in RBD + NAbs association. The SMD was used to generate US windows (cf. the ESI file). The free energy profile was then calculated using the WHAM. ${ }^{32}$ The binding free energy $\Delta G_{\mathrm{b}}$ between RBD and NAbs is able to calculate via PMF curve as mentioned in Figure 1E. ${ }^{30,} 31$ Moreover, the free energy barriers $\Delta G_{\text {on }}^{++}$and $\Delta G_{\text {off }}^{++}$, which were associated with the binding kinetic rate constant $k_{\text {on }}$ and the unbinding kinetic rate constant $k_{\text {off }}$ can be also estimated, respectively.
The calculated results for free energy barriers (cf. Table 1) indicated that the NAbs will bind to 501Y.V2 RBD more difficult than WT one because of the larger $\Delta G_{\text {on }}^{++}$.NAbs are much easier to bind to than to unbind from RBD, because the $\Delta G_{\text {off }}^{++}$is larger than the $\Delta G_{\text {on }}^{++}$. However, in the $\mathbf{M} 3$ case, the $\Delta G_{\text {off }}^{++}=0.14 \pm 0.18 \mathrm{kcal} \mathrm{mol}^{-1}$ is significantly smaller than the $\Delta G_{\text {on }}^{++}=2.83 \pm 0.65 \mathrm{kcal}^{\mathrm{mol}}{ }^{-1}$ indicating that it takes more time for NAb to bind to 501Y.V2 RBD for them unbind. Moreover, the observations were also confirmed due to the binding free energy, $\Delta G_{\mathrm{b}}$, calculations, in which the thermodynamic metric corresponding to the association between NAbs and RBD is significantly decreased when the 501Y.V2 variant was induced (Table 1). The NAb is thus resisted to bind to 501Y.V2 RBD. Therefore, it may be argued that the 501Y.V2 variant could reduce the vaccine efficiency. The observation is in good agreement with the experimental data. ${ }^{8,10,11,14}$ 
Table 1. The calculated results using SMD and US simulations. ${ }^{a}$

\begin{tabular}{lllcccc}
\hline N $^{0}$ & System & \multicolumn{1}{c}{$\boldsymbol{F}_{\text {Max }}$} & $\boldsymbol{W}$ & $\Delta \boldsymbol{G}_{\text {on }}^{++}$ & $\Delta \boldsymbol{G}_{\text {off }}^{++}$ & $\Delta \boldsymbol{G}_{\mathbf{b}}$ \\
\hline 1 & WT RBD + fNAb (w1) & $1388.0 \pm 18.6$ & $139.9 \pm 3.3$ & $0.24 \pm 0.20$ & $18.31 \pm 0.82$ & $-18.07 \pm 0.84$ \\
2 & 501Y.V2 RBD + fNAb (m1) & $859.3 \pm 40.7$ & $72.8 \pm 3.4$ & $0.81 \pm 0.26$ & $12.00 \pm 0.82$ & $-11.19 \pm 0.77$ \\
3 & 501Y.V2 RBD + fNAb (m2) & $1007.4 \pm 31.2$ & $86.8 \pm 3.1$ & $0.73 \pm 0.11$ & $11.62 \pm 0.54$ & $-10.89 \pm 0.55$ \\
4 & WT RBD + NAb (W1) & $1133.6 \pm 39.0$ & $178.8 \pm 8.5$ & $0.36 \pm 0.75$ & $39.82 \pm 1.31$ & $-39.46 \pm 1.08$ \\
5 & WT RBD + NAb (W2) & $1137.6 \pm 25.8$ & $181.5 \pm 6.5$ & $0.76 \pm 0.29$ & $43.36 \pm 0.73$ & $-42.60 \pm 0.67$ \\
6 & 501Y.V2 RBD + NAb (M1) & $745.5 \pm 25.4$ & $96.1 \pm 3.6$ & $0.62 \pm 0.15$ & $21.64 \pm 0.66$ & $-20.93 \pm 0.68$ \\
7 & 501Y.V2 RBD + NAb (M2) & $748.0 \pm 33.1$ & $81.6 \pm 6.5$ & $0.42 \pm 0.24$ & $16.16 \pm 0.88$ & $-15.74 \pm 0.91$ \\
8 & 501Y.V2 RBD + NAb (M3) & $470.2 \pm 25.7$ & $50.5 \pm 4.5$ & $2.83 \pm 0.65$ & $0.14 \pm 0.18$ & $-2.70 \pm 0.68$ \\
\hline
\end{tabular}

aThe calculated results over SMD and US simulations. The details of free energy profile and histograms over US simulations were reported in Figure $\mathrm{S}_{4}-\mathrm{S}_{7}$ of the ESI file.

The collective-variable $\mathrm{FEL}{ }^{45}$ was constructed by number of contacts between two proteins within $0.45 \mathrm{~nm}$ and the displacement of the antibody, revealed the unbinding pathway of NAbs. The obtained FEL was shown in Figure 5 and Figure S8 of the ESI file. The representative structures of the complexes within a backbone RMSD of $0.2 \mathrm{~nm}$ were then estimated using clustering method. ${ }^{33}$ The unbinding pathways were significantly altered under effects of the 501Y.V2 variant. A larger number of transition states of the WT RBD + fNAb complex implies that it is hard to unbind the antibody from
WT system than 501Y.V2 variant. Moreover, the representative structures $\mathbf{B}, \mathbf{b}$, and $\mathbf{b}^{\prime}$ correspond to the binding model of the RBD + fNAb complexes. The structures $\mathbf{D 7}, \mathbf{d} \mathbf{6}$, and $\mathbf{d} \mathbf{4}^{\prime}$ respond to the minima where the fNAb completely detached from RBD. The other conformations correspond to dissociated structures along unbinding pathways. The similar picture was also observed when the RBD + NAb complexes were investigated (Figure S8 of the ESI file).

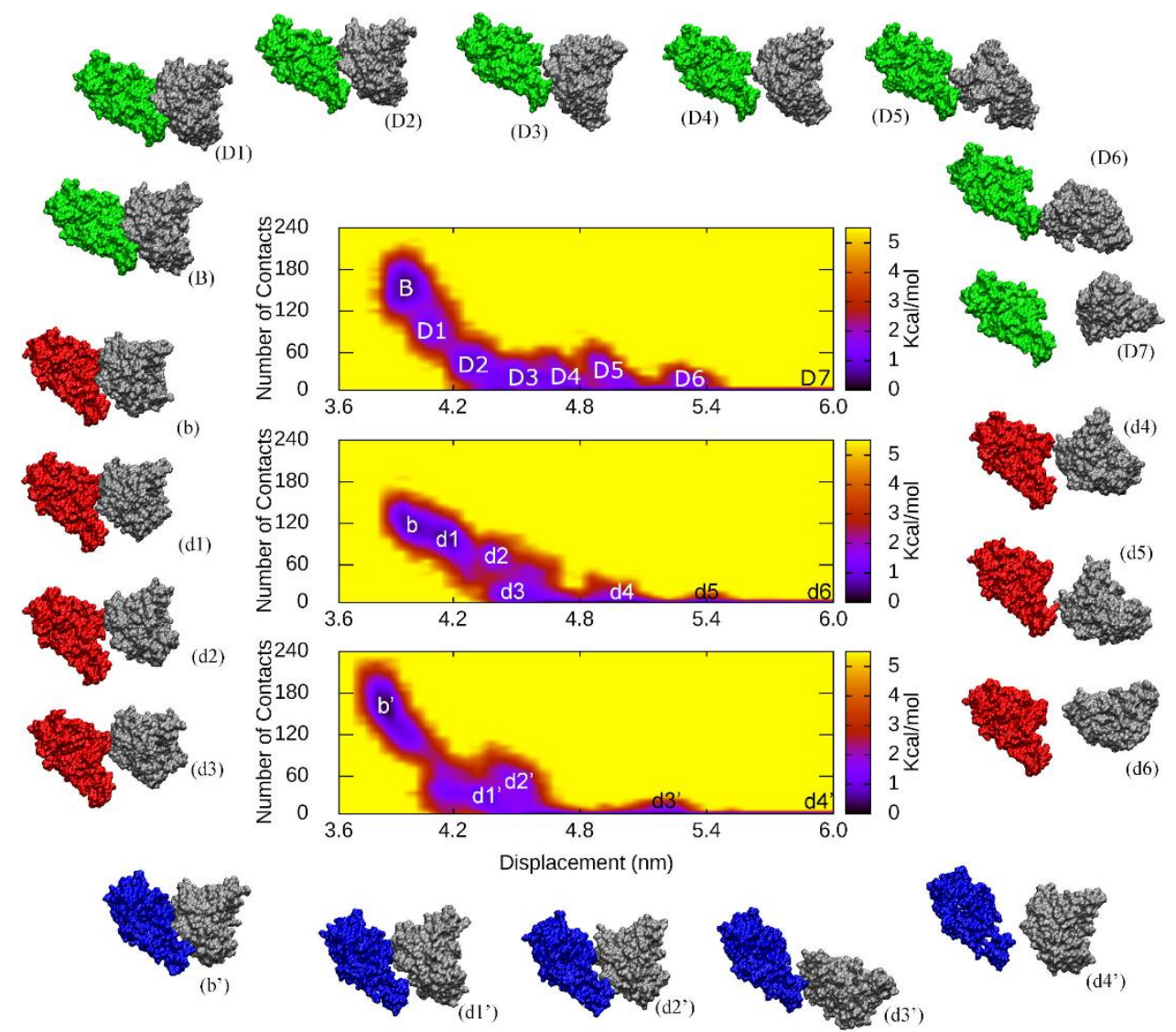

Figure 5. The collective-variable FEL revealed the unbinding pathways of fNAb from the binding mode with WT/501Y.V2 RBD. The representative structures of complexes were also estimated. 


\section{Conclusions}

In this work, the NAb resistance of 501Y.V2 variant was investigated using atomistic simulations. In particular, the binding pose of NAb/fNAb to WT/501Y.V2 RBD was revealed using atomistic simulations. Reducing number of $\mathrm{HB}$ and NB contacts between RBD and antibodies were observed when the 501Y.V2 variant was induced. Increasing FEL minima of 501Y.V2 RBD + NAb/fNAb in comparison with the WT RBD systems infer that the complex 501Y.V2 $\mathrm{RBD}+\mathrm{NAb} / \mathrm{fNAb}$ is more unstable than the WT one. Moreover, thermodynamics and kinetics of the binding process between RBD and NAb were also determined using SMD/US simulations. Interestingly, the binding free energy $\Delta G_{\mathrm{b}}$ of WT RBD + NAb/fNAb is significantly smaller than that of 501Y.V2 RBD + NAb/fNAb. It is consistent with results of the binding kinetic rate constant $k_{\text {on }}$ and the unbinding kinetic rate constant $k_{\text {off }}$. Poorly binding affinity of $\mathrm{NAb} / \mathrm{fNAb}$ to 501Y.V2 RBD confirm the antibody resistance of the South African variant. 8, 10,11, 14 Furthermore, the RBD + fNAb system can be used as an affordable model to investigate the change of the binding process between mutations RBD and antibodies. The required computing resources is thus reduced significantly.

\section{Acknowledgements}

This work was supported by Vietnam National Foundation for Science \& Technology Development (NAFOSTED) grant \#104.992019.57.

\section{References}

C. O. Barnes, A. P. West, K. E. Huey-Tubman, M. A. G. Hoffmann, N. G. Sharaf, P. R. Hoffman, N. Koranda, H. B. Gristick, C. Gaebler, F. Muecksch, J. C. C. Lorenzi, S. Finkin, T. Hägglöf, A. Hurley, K. G. Millard, Y. Weisblum, F. Schmidt, T. Hatziioannou, P. D. Bieniasz, M. Caskey, D. F. Robbiani, M. C. Nussenzweig and P. J. Bjorkman, Cell, 2020, 182, 828842.e816.

2. E. de Wit, N. van Doremalen, D. Falzarano and V. J. Munster, Nature Reviews Microbiology, 2016, 14, 523-534.

3. worldometrics, COVID-19 Coronavirus Pandemic, https://www.worldometers.info/coronavirus/.

4. S. T. Ngo, N. Quynh Anh Pham, L. Thi Le, D.-H. Pham and V. V. Vu, Journal of Chemical Information and Modeling, 2020, 60, 5771-5780.

5. D. Schoeman and B. C. Fielding, Virology Journal, 2019, 16, 69.

6. M. Hoffmann, H. Kleine-Weber, S. Schroeder, N. Kruger, T. Herrler, S. Erichsen, T. S. Schiergens, G. Herrler, N. H. Wu, A. Nitsche, M. A. Muller, C. Drosten and S. Pohlmann, Cell, 2020.

7. J. Lan, J. Ge, J. Yu, S. Shan, H. Zhou, S. Fan, Q. Zhang, X. Shi, Q. Wang, L. Zhang and X. Wang, Nature, 2020, 581, 215220.

8. C. K. Wibmer, F. Ayres, T. Hermanus, M. Madzivhandila, P. Kgagudi, B. Oosthuysen, B. E. Lambson, T. de Oliveira, M. Vermeulen, K. van der Berg, T. Rossouw, M. Boswell, V. Ueckermann, S. Meiring, A. von Gottberg, C. Cohen, L. Morris, J. N. Bhiman and P. L. Moore, Nature Medicine, 2021.

9. WHO, 2020.

10. P. Wang, M. S. Nair, L. Liu, S. Iketani, Y. Luo, Y. Guo, M. Wang, J. Yu, B. Zhang, P. D. Kwong, B. S. Graham, J. R. Mascola, J. Y. Chang, M. T. Yin, M. Sobieszczyk, C. A.
Kyratsous, L. Shapiro, Z. Sheng, Y. Huang and D. D. Ho, Nature, 2021.

11. M. Hoffmann, P. Arora, R. Groß, A. Seidel, B. F. Hörnich, A. S. Hahn, N. Krüger, L. Graichen, H. Hofmann-Winkler, A. Kempf, M. S. Winkler, S. Schulz, H.-M. Jäck, B. Jahrsdörfer, H. Schrezenmeier, M. Müller, A. Kleger, J. Münch and S. Pöhlmann, Cell, 2021.

12. Y. Weisblum, F. Schmidt, F. Zhang, J. DaSilva, D. Poston, J. C. C. Lorenzi, F. Muecksch, M. Rutkowska, H.-H. Hoffmann, E. Michailidis, C. Gaebler, M. Agudelo, A. Cho, Z. Wang, A. Gazumyan, M. Cipolla, L. Luchsinger, C. D. Hillyer, M. Caskey, D. F. Robbiani, C. M. Rice, M. C. Nussenzweig, T. Hatziioannou and P. D. Bieniasz, eLife, 2020, 9, e61312.

13. S. Jangra, C. Ye, R. Rathnasinghe, D. Stadlbauer, $H$. Alshammary, A. A. Amoako, M. H. Awawda, K. F. Beach, M. C. Bermúdez-González, R. L. Chernet, L. Q. Eaker, E. D. Ferreri, D. L. Floda, C. R. Gleason, G. Kleiner, D. Jurczyszak, J. C. Matthews, W. A. Mendez, L. C. F. Mulder, K. T. Russo, A.-B. T. Salimbangon, M. Saksena, A. S. Shin, L. A. Sominsky, K. Srivastava, F. Krammer, V. Simon, L. Martinez-Sobrido, A. García-Sastre and M. Schotsaert, The Lancet Microbe, 2021.

14. S. Cele, I. Gazy, L. Jackson, S.-H. Hwa, H. Tegally, G. Lustig, J. Giandhari, S. Pillay, E. Wilkinson, Y. Naidoo, F. Karim, Y. Ganga, K. Khan, M. Bernstein, A. B. Balazs, B. I. Gosnell, W. Hanekom, M.-Y. S. Moosa, R. J. Lessells, T. de Oliveira, A. Sigal, S. A. Ngs and C.-K. Team, Nature, 2021.

15. M. A. Tortorici, M. Beltramello, F. A. Lempp, D. Pinto, H. V. Dang, L. E. Rosen, M. McCallum, J. Bowen, A. Minola, S. Jaconi, F. Zatta, A. De Marco, B. Guarino, S. Bianchi, E. J. Lauron, H. Tucker, J. Zhou, A. Peter, C. Havenar-Daughton, J. A. Wojcechowskyj, J. B. Case, R. E. Chen, H. Kaiser, M. Montiel-Ruiz, M. Meury, N. Czudnochowski, R. Spreafico, J. Dillen, C. Ng, N. Sprugasci, K. Culap, F. Benigni, R. Abdelnabi, S.-Y. C. Foo, M. A. Schmid, E. Cameroni, A. Riva, A. Gabrieli, M. Galli, M. S. Pizzuto, J. Neyts, M. S. Diamond, H. W. Virgin, G. Snell, D. Corti, K. Fink and D. Veesler, Science, 2020, 370, 950-957.

16. L. Piccoli, Y.-J. Park, M. A. Tortorici, N. Czudnochowski, A. C. Walls, M. Beltramello, C. Silacci-Fregni, D. Pinto, L. E. Rosen, J. E. Bowen, O. J. Acton, S. Jaconi, B. Guarino, A. Minola, F. Zatta, N. Sprugasci, J. Bassi, A. Peter, A. De Marco, J. C. Nix, F. Mele, S. Jovic, B. F. Rodriguez, S. V. Gupta, F. Jin, G. Piumatti, G. Lo Presti, A. F. Pellanda, M. Biggiogero, M. Tarkowski, M. S. Pizzuto, E. Cameroni, C. Havenar-Daughton, M. Smithey, D. Hong, V. Lepori, E. Albanese, A. Ceschi, E. Bernasconi, L. Elzi, P. Ferrari, C. Garzoni, A. Riva, G. Snell, F. Sallusto, K. Fink, H. W. Virgin, A. Lanzavecchia, D. Corti and D. Veesler, Cell, 2020, 183, 1024-1042.e1021.

17. L. Casalino, Z. Gaieb, J. A. Goldsmith, C. K. Hjorth, A. C. Dommer, A. M. Harbison, C. A. Fogarty, E. P. Barros, B. C. Taylor, J. S. McLellan, E. Fadda and R. E. Amaro, ACS Cent. Sci., 2020, 6, 1722-1734.

18. H. Woo, S.-J. Park, Y. K. Choi, T. Park, M. Tanveer, Y. Cao, N. R. Kern, J. Lee, M. S. Yeom, T. I. Croll, C. Seok and W. Im, J. Phys. Chem. B, 2020, 124, 7128-7137.

19. B. Turoňová, M. Sikora, C. Schürmann, W. J. H. Hagen, S. Welsch, F. E. C. Blanc, S. von Bülow, M. Gecht, K. Bagola, C. Hörner, G. van Zandbergen, J. Landry, N. T. D. de Azevedo, S. Mosalaganti, A. Schwarz, R. Covino, M. D. Mühlebach, G. Hummer, J. Krijnse Locker and M. Beck, Science, 2020, 370, 203. 

B. Zhou, S. Song, X. Tang, J. Yu, J. Lan, J. Yuan, H. Wang, J. Zhao, S. Zhang, Y. Wang, X. Shi, L. Liu, J. Zhao, X. Wang, Z. Zhang and L. Zhang, Nature, 2020, 584, 115-119.

21. P. Schrödinger LLC, The PyMOL Molecular Graphics System, Version 1.3r1, 2010.

22. M. J. Abraham, T. Murtola, R. Schulz, S. Páll, J. C. Smith, B. Hess and E. Lindahl, SoftwareX, 2015, 1-2, 19-25.

23. A. E. Aliev, M. Kulke, H. S. Khaneja, V. Chudasama, T. D. Sheppard and R. M. Lanigan, Proteins: Struct., Funct., Bioinf., 2014, 82, 195-215.

24. H. Zhang, C. Yin, Y. Jiang and D. van der Spoel, J. Chem. Inf. Model., 2018, 58, 1037-1052.

25. H. Zhang, Y. Jiang, Z. Cui and C. Yin, J. Chem. Inf. Model., 2018, 58, 1669-1681.

26. W. L. Jorgensen, J. Chandrasekhar, J. D. Madura, R. W. Impey and M. L. Klein, J. Chem. Phys., 1983, 79, 926-935.

$27 . \quad$ S. T. Ngo, H. M. Hung, D. T. Truong and M. T. Nguyen, Phys. Chem. Chem. Phys., 2017, 19, 1909-1919.

28. N. T. Tung, P. Derreumaux, V. V. Vu, P. C. Nam and S. T. Ngo, ACS Omega, 2019, 4, 11066-11073.

29. T. Darden, D. York and L. Pedersen, J. Chem. Phys., 1993, 98, 10089-10092.

30. S. T. Ngo, K. B. Vu, L. M. Bui and V. V. Vu, ACS Omega, 2019, 4, 3887-3893.

31. S. T. Ngo, J. Comput Chem, 2021, 42, 117-123.

32. J. S. Hub, B. L. de Groot and D. van der Spoel, J. Chem. Theory Comput., 2010, 6, 3713-3720.

33. E. Papaleo, P. Mereghetti, P. Fantucci, R. Grandori and L. De Gioia, J Mol Graph Model, 2009, 27, 889-899.

34. B. Efron, Ann. Stat., 1979, 7, 1-26.

35. A. C. Pan, D. Jacobson, K. Yatsenko, D. Sritharan, T. M. Weinreich and D. E. Shaw, Proc Natl Acad Sci U S A, 2019, 116, 4244-4249.

36. P. J. Lupardus, M. Ultsch, H. Wallweber, P. Bir Kohli, A. R. Johnson and C. Eigenbrot, Proc. Natl. Acad. Sci. U.S.A, 2014, 111, 8025-8030.

37. Y. Shan, K. Gnanasambandan, D. Ungureanu, E. T. Kim, H. Hammarén, K. Yamashita, O. Silvennoinen, D. E. Shaw and S. R. Hubbard, Nat. Struct. Mol. Biol., 2014, 21, 579-584.

38. C. Tang, J. Iwahara and G. M. Clore, Nature, 2006, 444, 383386.

39. C. Frisch, A. R. Fersht and G. Schreiber, J. Mol. Biol., 2001, 308, 69-77.

40. F. Chen, H. Liu, H. Y. Sun, P. C. Pan, Y. Y. Li, D. Li and T. J. Hou, Phys Chem Chem Phys, 2016, 18, 22129-22139.

41. M. Sikora, S. von Bülow, F. E. C. Blanc, M. Gecht, R. Covino and G. Hummer, PLoS Comput. Biol., 2021, 17, e1008790.

42. S. T. Ngo, D. T. Truong, N. M. Tam and M. T. Nguyen, J. Mol. Graph. Model., 2017, 76, 1-10.

43. J. A. Lemkul and D. R. Bevan, J Phys Chem. B, 2010, 114, 1652-1660.

44. Y. Mu, P. H. Nguyen and G. Stock, Proteins: Struct., Funct., Bioinf., 2005, 58, 45-52.

45. T. H. Nguyen, G. Rossetti, F. Arnesano, E. Ippoliti, G. Natile and P. Carloni, J. Chem. Theor. Compt, 2014, 10, 35783584 . 